\title{
Cofilin-1 as a potential biomarker for Mycobacterium tuberculosis infection
}

\author{
YIPING XIE $^{1 *}$, ZHIQIN ZHANG $^{2 *}$, MIN ZHANG $^{1}$ and $\mathrm{HUI} \mathrm{CAO}^{3}$ \\ ${ }^{1}$ Department of Clinical Laboratory, Affiliated Kunshan Hospital of Jiangsu University; ${ }^{2}$ Biological Sample Bank, \\ Affiliated Kunshan Hospital of Jiangsu University, Kunshan, Jiangsu 215300; ${ }^{3}$ Department of Food Safety and Evaluation, \\ Jiangsu Provincial Center for Disease Control and Prevention, Nanjing, Jiangsu 210009, P.R. China
}

Received October 12, 2021; Accepted January 5, 2022

DOI: $10.3892 /$ etm.2022.11178

\begin{abstract}
Tuberculosis (TB) induced by Mycobacterium tuberculosis $(M . t b)$, is one of the deadliest human infections worldwide. Our previous studies demonstrated cofilin-1 (CFL1) expression was significantly increased in exosomes from Mycobacterium avium (M. avium)-infected macrophages. The expression of CFL1 protein in $M$. $t b$ infected hosts was investigated in the present study to predict whether CFL1 could have potential as a biomarker for $M$. $t b$ infection. In the present study, the mRNA and protein expression levels of CFL1 in M. avium-infected macrophages and supernatants were analyzed via reverse transcription-quantitative PCR and western blotting. Furthermore, CFL1 expression in macrophages was knocked down in vivo, and then CFL1 expression levels in $M$. avium-infected macrophages and supernatant were detected via western blotting and ELISA. In addition, CFL1 was detected in the peripheral blood mononuclear cells and plasma of patients with TB using western blotting and ELISA. The specificity and sensitivity of CFL1 as a biomarker and the association between TB infection and normal individuals were compared and analyzed using GraphPad Prism 5. CFL1 protein expression levels were significantly increased in $M$. avium-infected macrophages and supernatant. Meanwhile, CFL1 was upregulated in patients with TB. Bioinformatics statistics indicated the high specificity and sensitivity of CFL1 in patients with TB. Thus, these results suggest that CFL1 may act as a potential biomarker of TB infection.
\end{abstract}

Correspondence to: Dr Hui Cao, Department of Food Safety and Evaluation, Jiangsu Provincial Center for Disease Control and Prevention, 172 Jiangsu Road, Gulou, Nanjing, Jiangsu 210009, P.R. China

E-mail:2534839279@qq.com

${ }^{*}$ Contributed equally

Key words: cofilin-1, macrophages, tuberculosis, biomarkers

\section{Introduction}

Tuberculosis (TB) induced by Mycobacterium tuberculosis, is one of the deadliest human infections worldwide (1). In 2021, there were 5.8 million new TB cases globally, and nearly 1.5 million patients died from TB-associated disease (2). Over the past 100 years, great achievements have been made in TB treatment and control through the Bacillus Calmette-Guerin vaccination and the application of anti-TB drugs (3). However, there is still a lack of understanding of the host-pathogen interactions that occur to better control TB (4). In view of the global TB infection rate of $1 / 3$, early TB recognition and treatment are urgently required (5). At present, clinical studies have uncovered that early TB diagnosis is still quite difficult, and the rates of morbidity and mortality are increased due to misdiagnosis (6-8).

Cofilin (CFL), an actin decomposing factor, is expressed in various cells and modulates the generation of actin filaments by controlling polymer formation and degradation (9). As the non-muscle isoform of its gene product, CFL1 is required by the importin-9-regulated nuclear migration of G-actin (10) and is involved in transcription extension modulated by RNA polymerase II (11). CFL1 was previously recognized as a critical protein in the routine nuclear structures and roles of remote cell types (12). CFL1 has an essential role in cell movement and cytoplasmic changes and is directly associated with the invasion, spread and drug resistance of various human malignancies such as mammary tumors (13-16). Of note, our previous proteomics studies on exosomes found that CFL1 was significantly increased in the supernatant of Mycobacterium avium infected macrophages (17) and was also upregulated in M. avium-infected macrophages (18).

The present study aimed to explore the expression of cofilin-1 in M. avium-infected macrophages and the blood of patients with TB and investigate the possibility of CFL1 as a marker for use in TB diagnosis.

\section{Materials and methods}

Patient sample preparation. Between May 2019 and July 2020 , a total of 36 patients with active TB (16 female; age, 25-65 years; mean age, 46.6 years; 20 males, age, 24-63 years, mean age, 43.6 years) and 34 healthy subjects (17 female; age, 
22-56 years; mean age, 40.2 years; 17 males, age, 20-55 years, mean age, 38.5 years) were recruited for the present study at The First People's Hospital of Kunshan Affiliated to Jiangsu University (Kunshan, China). EDTA-treated peripheral blood $(5 \mathrm{ml})$ and plasma were collected from all subjects. Approval was obtained from the Ethics and Scientific Committee of the Affiliated Kunshan Hospital of Jiangsu University, and all participants signed an informed consent form (approval no. IEC-C-007-A07-V3.0). The experiments were performed in accordance with the Declaration of Helsinki Principles. TB patients were diagnosed according to the clinical criteria from the WHO (19), and normal individuals included those with the results of negative tuberculin skin tests.

Peripheral blood $(5 \mathrm{ml})$ treated with EDTA was mixed with PBS at a 1:1 ratio and added into $50 \mathrm{ml}$ centrifuge tubes, to which $20 \mathrm{ml}$ Ficoll separation solution was added, and centrifugation was performed at $1,200 \mathrm{x}$ g for $30 \mathrm{~min}$ at room temperature. Finally, peripheral blood mononuclear cells (PBMCs) were transferred into new tubes and stored at $-80^{\circ} \mathrm{C}$ for the subsequent experiments.

Cell-free plasma was isolated from all blood samples using heparin anticoagulant tubes, and centrifuged at $4,000 \mathrm{x} \mathrm{g}$ for $30 \mathrm{~min}$ at room temperature. The plasma samples were then separated into microcentrifuge tubes and stored at $-80^{\circ} \mathrm{C}$.

Cell culture. THP-1 cells (The Cell Bank of the Type Culture Collection of The Chinese Academy of Sciences) were cultured in Dulbecco's modified Eagle's medium (DMEM) (Hyclone; GE Healthcare, USA) with $10 \%$ fetal bovine serum (FBS) (Hyclone; GE Healthcare) and $1 \%$ penicillin-streptomycin, and cultured at $37^{\circ} \mathrm{C}$ in a humid condition with $5 \% \mathrm{CO}_{2}$. THP-1 cells grown in 6-well plates at $75 \%$ confluence were induced into macrophages with $0.1 \mathrm{nM}$ phorbol ester. Macrophages were infected with M. avium (Mol 1:10, 1:50, 1:100, 1:200) for $24 \mathrm{~h}$.

Cell transfection. Small interfering RNA (siRNA) of CFL1 $(50 \mathrm{~nm})$ (Shanghai GenePharma Co., Ltd.) were transfected into macrophages grown in 6-well plates at $75 \%$ confluence using Lipofectamine ${ }^{\circledR} 3000$ reagent (Invitrogen; Thermo Fisher Scientific, Inc.) at $37^{\circ} \mathrm{C}$ for $24 \mathrm{~h}$ according to the manufacturer's instructions. The sequences used were as follows: CFL1 siRNA sense, 5'-GAAGGUGCGUAAGUCUUCATT-3' and antisense, 5'-UGAAGACUUACGCACCUUCTT-3'; and scrambled siRNA sense, 5'-AUAUUCCUGCGAUAGCUC GTT-3' and antisense, 5'-CGAGCUAUCGCAGGAAUA UTT-3'.

Reverse transcription-quantitative PCR (RT-qPCR). Total RNA was extracted using TRIzol ${ }^{\circledR}$ reagent (Invitrogen; Thermo Fisher Scientific, Inc.) according to the manufacturer's instructions. cDNA was synthesized from isolated RNA with a PrimeScript ${ }^{\mathrm{TM}}$ RT reagent kit (Takara Biotechnology Co., Ltd.). qPCR was performed with the Super SYBR Green kit (Thermo Fisher Scientific, Inc.) on ABI7500 (Thermo Fisher Scientific, Inc.) instrument. The qPCR primers were as follows: CFL1-forward (F), 5'-TGCCCTCTCCTTTTCGTTTCC-3' and -reverse (R), 5'-CTCCTCTGGCGTTGAAGACT-3'; and GAPDH-F, 5'-AGAAGGCTGGGGCTCATTTG-3' and -R, 5'-AGGGGCCATCCACAGTCTTC-3'. The thermocycling conditions were: $95^{\circ} \mathrm{C}$ for $10 \mathrm{~min}$, followed by 36 cycles of $95^{\circ} \mathrm{C}$ for $25 \mathrm{sec}$ and $60^{\circ} \mathrm{C}$ for $30 \mathrm{sec}$. The mRNA fold-change was calculated by using the $2^{-\Delta \Delta C q}$ method (20).

Cytokine analysis. Macrophages cultured in 6-well plates were treated with M. avium or transfected with CFL1-siRNA for $24 \mathrm{~h}$. The expression of CFL1 in the plasma and supernatant of transfected cells of patients with TB was detected by ELISA (cat. no. CSB-EL005280HU, Cusabio Technology LLC). The cytokine content was determined by the standard curve method.

Western blotting. The PBMCs and transfected cells were lysed with RIPA lysate (cat. no: P0013C, Beyotime Biotechnology Corp.) for BCA protein quantification. The proteins $(20 \mu \mathrm{g})$ were separated via SDS-PAGE on $10 \%$ gel and subsequently transferred onto a PVDF membrane (Bio-Rad Laboratories, Inc.). The membranes were first incubated with primary anti-CFL1 antibodies (cat. no. CSB-PA001739; 1:1,200, Cusabio Technology LLC) at $37^{\circ} \mathrm{C}$ for $60 \mathrm{~min}$ and then with HRP-conjugated Affinipure goat anti-rabbit $\operatorname{IgG}(\mathrm{H}+\mathrm{L})$ secondary antibody at $37^{\circ} \mathrm{C}$ for $120 \mathrm{~min}$. After washing, immunoreactivity was visualized using Chemiluminescent Substrate System (Thermo Fisher Scientific, Inc.) according to the instructions of the manufacturer. Band intensity was evaluated using ImageJ 1.45 software (National Institutes of Health).

Receiver operating characteristic (ROC) curves. The diagnostic ability of CFL1 in patients infected with TB was analyzed by ROC curves. The area under the ROC curve (AUC) detects the capability of an index to differentiate two groups. AUCs of $90-100 \%, 80-90 \%, 70-80 \%, 60-70 \%$, $50-60 \%$ and $<50 \%$ generally imply excellent, good, fair, poor, bad (or failed) and incapable (or random data), respectively. Therefore, the ROC curve was used to evaluate the predictive power of CFL1 in discriminating between healthy individuals and patients with TB.

Statistical analysis. All statistical tests were performed using GraphPad Prism 5 (GraphPad Software, Inc.). One-way ANOVA followed by Tukey's post hoc test were performed on this statistical software and the statistical test used is mentioned in the figure legends. All experimental results are expressed as the mean \pm SEM from three individual experiments. $\mathrm{P}<0.05$ was considered to indicate a statistically significant difference.

\section{Results}

M. avium infection promotes the expression of CFL1 in macrophages. ELISA, RT-qPCR and western blotting were performed to detect the expression of CFL1 mRNAs and proteins in the supernatant of $M$. avium infected macrophages or infected cells. CFL1 mRNA was significantly elevated in the macrophages as determined by RT-qPCR (Fig. 1A). Moreover, the results of ELISA showed that CFL1 protein was significantly enhanced in $M$. avium-infected macrophages (Fig. 1B). In addition, CFL1 protein were also significantly elevated in M. avium-infected macrophages as determined by western blotting (Fig. 1C and D). 
A

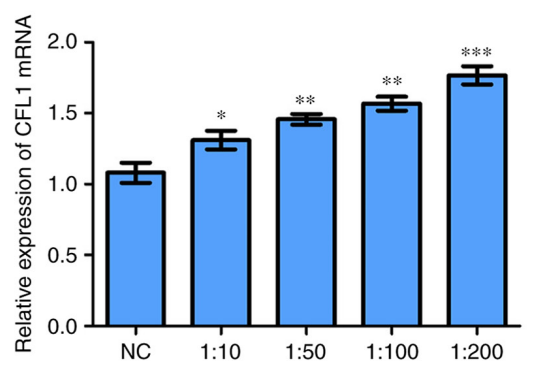

C

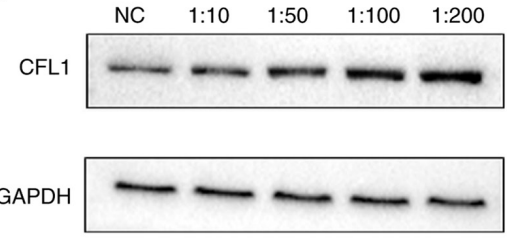

B

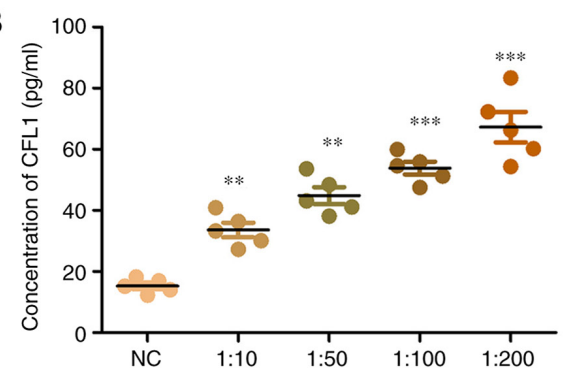

D

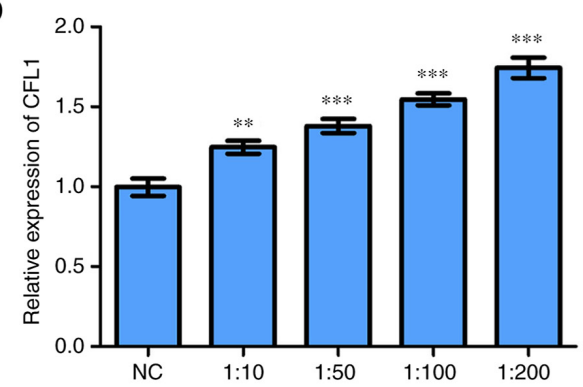

Figure 1. Expression of CFL1 in M. avium-infected macrophages. (A) Expression of CFL1 mRNA in macrophages infected with M. avium as determined via reverse transcription-quantitative PCR. (B) The concentration of CFL1 in the supernatant of macrophages infected with $M$. avium was measured by ELISA. (C and D) The expression levels of CFL1 protein in macrophages were detected via western blotting. ${ }^{*} \mathrm{P}<0.05,{ }^{* * *} \mathrm{P}<0.005,{ }^{* * * *} \mathrm{P}<0.001$ vs. NC. M. avium, Mycobacterium avium. NC, negative control; CFL1, cofilin-1.

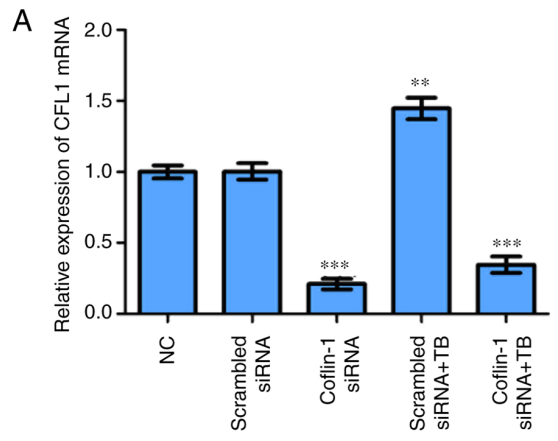

C

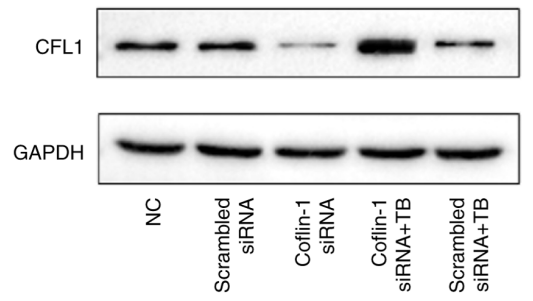

B

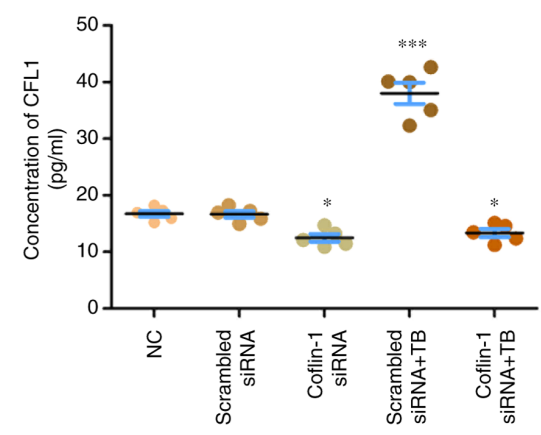

D

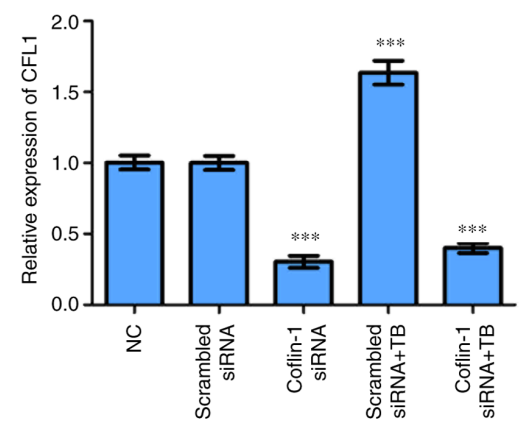

Figure 2. Expression of CFL1 in macrophages is influenced by M. avium infection (TB). (A) Expression of CFL1 mRNA in macrophages was regulated by CFL1 and $M$. avium (TB) as determined via reverse transcription-quantitative PCR. (B) CFL1 protein expression in the supernatant of M. avium-infected macrophages as measured by ELISA. (C and D) CFL1 protein expression levels in CFL1-siRNA transfected macrophages or M. avium-infected cells were detected via western blotting. Scrambled siRNA, cofilin-1 siRNA, scrambled siRNA + TB, cofilin- 1 siRNA vs. NC. "P<0.05, ${ }^{* *} \mathrm{P}<0.005,{ }^{* * *} \mathrm{P}<0.001$ vs. NC. NC, normal control; M. avium, Mycobacterium avium; siRNA, small interfering RNA; CFL1, cofilin-1; TB, tuberculosis.

M. avium promotes the expression of CFL1 in macrophages. To explore the effect of $M$. avium on CFL1 expression in macrophages, CFL1 siRNA was transfected into macrophages. It was observed that $C F L 1$ mRNA expression levels were significantly decreased in cells and $M$. avium-infected macrophages transfected with siRNA compared with the controls, whereas $M$. avium enhanced CFL1 expression in siRNA-transfected cells compared with the scrambled siRNA or NC groups (Fig. 2A). In addition, CFL1 protein expression was increased in the supernatant and cells stimulated with 
A

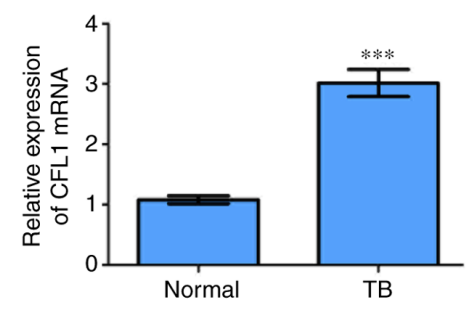

D
B

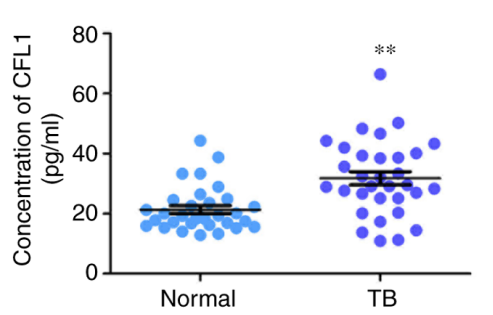

C

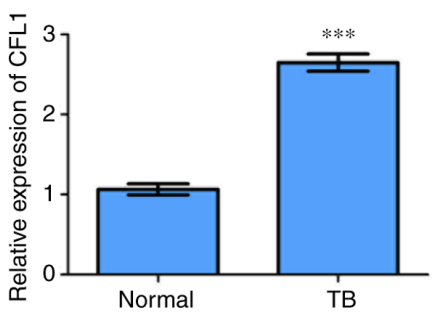

E
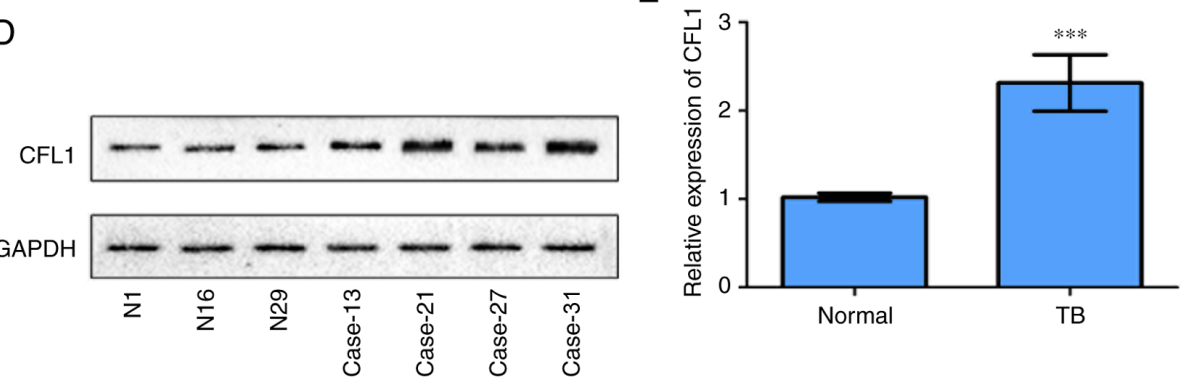

Figure 3. Expression levels of CFL1 in patients infected with M. avium infection (TB) were quantified. (A) CFL1 mRNA expression levels in the PBMCs of patients infected with TB were detected via reverse transcription-quantitative PCR. (B) The concentration of CFL1 protein in the plasma of patients infected with TB was measured by ELISA. (C) The relative expression of CFL1 protein in PBMCs of patients infected with TB was determined via western blotting. ${ }^{* *} \mathrm{P}<0.005,{ }^{* * *} \mathrm{P}<0.001$. vs. Normal cases. (D and E) Partial results of western blotting experiments of CFL1 protein in PBMCs of patients infected with TB were performed and the bands were statistically analyzed. TB cases (13\#, 21\#, 27\#, and 31\#) vs. normal cases (N, $1 \#, 16 \#, 29 \#) .{ }^{* * *} \mathrm{P}<0.001 \mathrm{vs.} \mathrm{Normal}$. TB, tuberculosis; PBMCs, peripheral blood mononuclear cells; CFL1, cofilin-1.

A

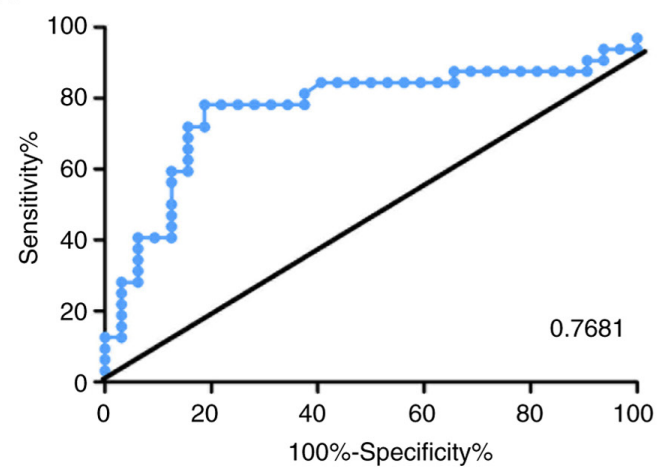

B

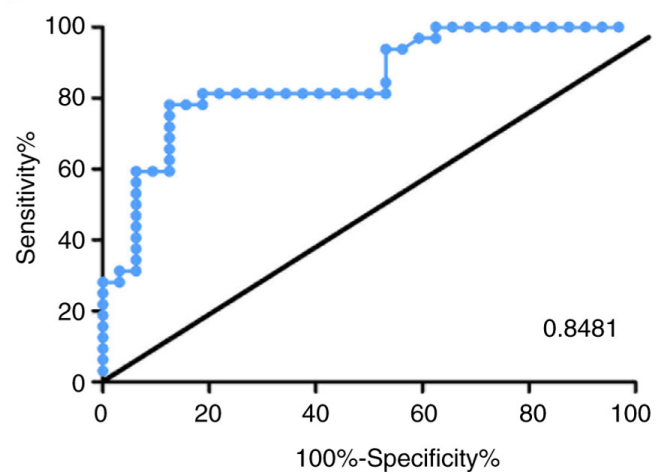

Figure 4. Receiver operating characteristic (ROC) curves show the diagnostic ability of CFL1 to distinguish patients with TB infection from healthy controls. (A) CFL1 mRNA expression in PBMCs to distinguish TB infection from healthy controls. (B) CFL1 protein expression in plasma to distinguish TB from healthy controls. AUC values between 90 and $100 \%$ are considered excellent, $80-90 \%$ good, $70-80 \%$ fair, $60-70 \%$ poor, $50-60 \%$ bad classifiers. Area under the ROC curve (AUC) $<50 \%$ indicates random values that are not capable of distinguishing between two groups. TB, tuberculosis; PBMCs, peripheral blood mononuclear cells; CFL1, cofilin-1.

M. avium compared with the scrambled siRNA or NC groups (Fig. 2B-D). These findings revealed that M. avium could promote CFL1 expression in macrophages.

CFL1 is highly expressed in patients infected with TB. In vitro experiments showed that infection with $M$. avium could promote the expression of CFL1. RT-qPCR results demonstrated that CFL1 mRNA expression was significantly increased in patients with TB compared with normal controls (Fig. 3A). In addition, as determined by ELISA, CFL1 protein was markedly elevated in both plasma and PBMCs from the TB patients compared with the normal patients (Fig. 3B and C). Western blotting results showed that the expression of CFL1 protein in the PBMCs of patients with
TB was significantly increased compared with the normal controls (Fig. 3D and E).

CFL1 may be an effective predictor of patients with $T B$ through ROC curve. ROC curve showed that CFL1 mRNA expression was highly effective in distinguishing patients with TB from healthy controls (AUC, 81.81\%; Fig. 4A and B).

\section{Discussion}

CFL1, a primary actin-linking factor in platelets, is required to sever actin filaments during reconstruction of the actin cytoskeleton (21). CFL1 is closely associated with tumor occurrence and can act as a diagnostic marker for several types of tumors (22). 
For instance, excessive CFL1 expression was found to inhibit the progression and spread of non-small cell lung cancer (23), and CFL phosphorylation was found to be higher in bladder cancer tissues compared with that observed in healthy bladder tissues (24). An accumulating number of reports have shown that CFL1 protein may mark the diagnosis and prognosis of tumors (25-27). For example CFL1 expression was found to be significantly increased in the blood of patients with lung adenocarcinoma compared with healthy individuals (28).

Moreover, previous literature suggests the significant roles of CFL1 in microbial infection-related diseases. For example, the activity of CFL1 following herpes simplex virus (HSV)-1 infection may be modulated by a RhoGTPase-regulated transduction pathway in the pathogenesis of HSV-1-induced neurological disorders (29). CFL1 has been reported to be upregulated in exosomes derived from $M$. avium-infected macrophages (17). In addition, infection by Mycobacterium tuberculosis (M. tb) regulates coronin1-mediated upregulation of intracellular cAMP, followed by activation of CFL1 and the depolymerization of F-actin to suppress phagosomal acidification and its maturation in macrophages (30).

In the present study, upregulated CFL1 gene and protein expression levels were found in $M$. avium-infected macrophages. Meanwhile, the knockdown experiments revealed that CFL1 expression was influenced by $M$. avium infection. The expression of CFL1 in macrophages detected by immunohistochemistry will be analyzed in future experiments.

In view of its numerous biological functions in tumor cells and its potential as a diagnostic marker, we speculated that CFL1 may also be used as a biomarker of TB infection. In the in vitro experiments, CFL1 was notably upregulated in 36 patients with TB compared with healthy individuals. The CFL1 mRNA and protein expression levels of AUC in patients with TB and controls were 0.84 and 0.76 , respectively indicating that they have good value as diagnostic markers. CFL1 expression showed diagnostic value in detecting TB infection.

Due to the limited number of samples, this was a preliminary study to explore the diagnostic value of CFL1 in the peripheral blood of patients with TB. In future studies, we will collect a large number of samples and add a sample group of patients with latent TB. In summary, the results of the present study indicated that CFL1 is a noteworthy target from the perspective of TB peripheral blood diagnosis, and our research team will continue to investigate it.

\section{Acknowledgements}

Not applicable.

\section{Funding}

This work was supported by grants from the Science and Technology Project of Suzhou (grant no. KJXW2018068) and the Medical Research Project of Jiangsu Health Commission (grant no. Z2019001).

\section{Availability of data and materials}

The datasets used and/or analyzed during the current study are available from the corresponding author on reasonable request.

\section{Authors' contributions}

$\mathrm{HC}$ and $\mathrm{YX}$ designed the present study. YX and ZZ performed the experiments and wrote the manuscript, and $\mathrm{MZ}$ analyzed the data and revised the manuscript. All authors read and approved the final manuscript, and confirm the authenticity of all the raw data.

\section{Ethics approval and consent to participate}

Approval for the protocol of the present study was obtained from the Ethics and Scientific Committee of the Affiliated Kunshan Hospital of Jiangsu University, and all participants signed an informed consent form (approval no. IEC-C-007-A07-V3.0).

\section{Patient consent for publication}

Not applicable.

\section{Competing interests}

The authors declare that they have no competing interests.

\section{References}

1. Wang J, Wang Y, Tang L and Garcia RC: Extracellular vesicles in mycobacterial infections: Their potential as molecule transfer vectors. Front Immunol 10: 1929, 2019.

2. Zhang M, Xie Y, Li S, Ye X, Jiang Y, Tang L and Wang J: Proteomics analysis of exosomes from patients with active tuberculosis reveals infection profiles and potential biomarkers. Front Microbiol 12: 800807, 2022.

3. Shimao T: Tuberculosis and its control-lessons from the past and future prospect. Kekkaku 80: 481-489, 2015 (In Japanese).

4. Dheda K, Barry CE III and Maartens G: Tuberculosis. Lancet 387: 1211-1226, 2016.

5. Dye C, Floyd K and Uplekar M: In global tuberculosis control: Surveillance, planning, financing. Vol. 1. Ch., pp17-37, 2008.

6. Cheon SA, Cho HH, Kim J, Lee J, Kim HJ and Park TJ: Recent tuberculosis diagnosis toward the end TB strategy. J Microbiol Methods 123: 51-61, 2016

7. Galimi R: Extrapulmonary tuberculosis: Tuberculous meningitis new developments. Eur Rev Med Pharmacol Sci 15: 365-386, 2011.

8. Furin J, Cox H and Pai M: Tuberculosis. Lancet 393: 1642-1656, 2019.

9. Vartiainen MK, Mustonen T, Mattila PK, Ojala PJ, Thesleff I, Partanen $\mathbf{J}$ and Lappalainen P: The three mouse actin-depolymerizing factor/cofilins evolved to fulfill cell-type-specific requirements for actin dynamics. Mol Biol Cell 13: 183-194, 2020.

10. Percipalle P: Co-transcriptional nuclear actin dynamics. Nucleus 4: 43-52, 2013.

11. Obrdlik A and Percipalle P: The F-actin severing protein cofilin-1 is required for RNA polymerase II transcription elongation. Nucleus 2: 72-79, 2011.

12. Wiggan O, Schroder B, Krapf D, Bamburg JR and DeLuca JG: Cofilin regulates nuclear architecture through a myosin-II dependent mechanotransduction module. Sci Rep 7: 40953, 2017.

13. Wang W, Mouneimne G, Sidani M, Wyckoff J, Chen X, Makris A, Goswami S, Bresnick AR and Condeelis JS: The activity status of cofilin is directly related to invasion, intravasation, and metastasis of mammary tumors. J Cell Biol 173: 395-404, 2006.

14. Ono S: Mechanism of depolymerization and severing of actin filaments and its significance in cytoskeletal dynamics. Int Rev Cytol 258: 1-82, 2007.

15. Hotulainen P, Paunola E, Vartiainen MK and Lappalainen P: Actin-depolymerizing factor and cofilin-1 play overlapping roles in promoting rapid F-actin depolymerization in mammalian nonmuscle cells. Mol Biol Cell 16: 649-664, 2005. 
16. Kapoor S: Cofilin-1 overexpression and its role in tumor growth and progression in systemic malignancies. Int J Radiat Biol 90: $113,2014$.

17. Wang JJ, Chen C, Xie PF, Pan Y, Tan YH and Tang LJ: Proteomic analysis and immune properties of exosomes released by macrophages infected with Mycobacterium avium. Microbes Infect 16: 283-291, 2014.

18. Wang J, Yao Y, Wu J, Deng Z, Gu T, Tang X, Cheng Y and Li G: The mechanism of cytoskeleton protein $\beta$-actin and cofilin- 1 of macrophages infected by Mycobacterium avium. Am J Transl Res 8: 1055-1063, 2016.

19. World Health Organization; International Union Against Tuberculosis and Lung Disease; Royal Netherlands Tuberculosis Association: Revised international definitions in tuberculosis control. Int J Tuberc Lung Dis 5: 213: 215, 2001

20. Livak KJ and Schmittgen TD: Analysis of relative gene expression data using real-time quantitative PCR and the 2(-Delta Delta C(T)) method. Methods 25: 402-408, 2001.

21. Dasgupta SK and Thiagarajan P: Cofilin-1-induced actin reorganization in stored platelets. Transfusion 60: 806-814, 2020.

22. Shishkin S, Eremina L, Pashintseva N, Kovalev L and Kovaleva M: Cofilin-1 and other ADF/cofilin superfamily members in human malignant cells. Int J Mol Sci 18: 10, 2016.

23. Tsai CH, Lin LT, Wang CY, Chiu YW, Chou YT, Chiu SJ, Wang HE, Liu RS, Wu CY, Chan PC, et al: Over-expression of cofilin-1 suppressed growth and invasion of cancer cells is associated with up-regulation of let-7 microRNA. Biochim Biophys Acta 1852: 851-861, 2015 .

24. Chung H, Kim B, Jung SH, Won KJ, Jiang X, Lee CK, Lim SD, Yang SK, Song KH and Kim HS: Does phosphorylation of cofilin affect the progression of human bladder cancer? BMC Cancer 13: 45,2013 .
25. Jiang N, Kham SK, Koh GS, Suang Lim JY, Ariffin H, Chew FT and Yeoh AE: Identification of prognostic protein biomarkers in childhood acute lymphoblastic leukemia (ALL). J Proteomics 74: 843-857, 2011

26. Peng XC, Gong FM, Zhao YW, Zhou LX, Xie YW, Liao HL, Lin HJ, Li ZY, Tang MH and Tong AP: Comparative proteomic approach identifies PKM2 and cofilin-1 as potential diagnostic, prognostic and therapeutic targets for pulmonary adenocarcinoma. PLoS One 6: e27309, 2011.

27. Guan M, Chen X, Ma Y, Tang L, Guan L, Ren X, Yu B, Zhang W and Su B: MDA-9 and GRP78 as potential diagnostic biomarkers for early detection of melanoma metastasis. Tumour Biol 36: 2973-2982, 2015

28. Zheng Y, Fang Y, Li S and Zheng B: Detection of plasma cofilin protein for diagnosis of lung cancer. Nan Fang Yi Ke Da Xue Xue Bao 33: 1551-1553, 2013 (In Chinese).

29. Xiang Y, Zheng K, Ju H, Wang S, Pei Y, Ding W, Chen Z, Wang Q, Qiu X, Zhong M, et al: Cofilin 1-mediated biphasic F-actin dynamics of neuronal cells affect herpes simplex virus 1 infection and replication. J Virol 86: 8440-8451, 2012.

30. Saha S, Hazra A, Ghatak D, Singh AV, Roy S and BoseDasgupta S: A bumpy ride of mycobacterial phagosome maturation: Roleplay of coronin1 through Cofilin1 and cAMP. Front Immunol 12: $687044,2021$.

This work is licensed under a Creative Commons Attribution-NonCommercial-NoDerivatives 4.0 International (CC BY-NC-ND 4.0) License. 